\title{
De l'ethos et du biographique : au croisement de deux parcours
}

\author{
Anne-Marie CLÉMENT \\ Université du Québec à Rimouski
}

Issue de la rhétorique ancienne, la notion d'ethos a connu au cours des dernières décennies un certain regain d'intérêt, si bien qu'elle est aujourd'hui réactualisée dans divers champs de la théorie contemporaine. À l'origine rattaché à l'art oratoire, l'ethos désigne la composante de l'argumentation qui correspond à l'image que le locuteur construit de lui-même dans son discours en vue d'inspirer confiance. Pour Aristote, l'ethos se rapporte uniquement à l'instance d'énonciation à l'intérieur du discours, mais il en sera autrement pour d'autres traditions comme celle de la rhétorique classique où l'ethos concerne la personne même de l'orateur, dépendant à la fois du 
statut social et des mœurs de ce dernier, se situant donc en dehors des limites du discours. Aujourd'hui, la pertinence de la notion déborde le strict domaine de l'éloquence et se trouve associée à divers champs disciplinaires tels que la linguistique de l'énonciation, l'analyse du discours, la rhétorique contemporaine, la sociologie des champs. Selon les approches, elle permet de prendre en compte la vocalité spécifique du texte qui suppose une source énonciative dont le lecteur doit construire la figure à partir de divers indices textuels; dans d'autres cas, elle permet de s'arrêter à la dimension discursive de la posture de l'écrivain, dans sa manière singulière d'occuper une position précise dans le champ littéraire ; ou, encore, elle se donne à saisir comme partie constituante de l'identité littéraire. Est ainsi reconduite la possibilité de considérer l'ethos selon une perspective discursive qui recherche l'efficacité de la parole à l'intérieur de l'échange verbal ou selon une perspective interactionnelle sociale et institutionnelle, donc extérieure au discours du locuteur. Les différentes approches peuvent être complémentaires, l'efficacité de la parole n'étant « ni purement extérieure (institutionnelle) ni purement interne (langagière) ", comme le précise Ruth Amossy à propos de l'approche à la fois pragmatique et sociologique de la rhétorique contemporaine qui permet ainsi « de travailler dans la matérialité du discours et d'analyser la construction de l'ethos en termes d'énonciation et de genre de discours » et « de souligner la dimension sociale de l'ethos discursif, mais aussi sa relation à des positions institutionnelles extérieures. »(1999, p. 147-148) Le corpus

1 Ruth Amossy propose une rétrospective des reformulations de la notion d'ethos dans l'introduction de l'ouvrage collectif Images de soi dans le discours. La construction de l'ethos (1999) paru sous sa direction. 
des textes examinés sous l'angle de la constitution de l'ethos ne se restreint pas à des écrits strictement argumentatifs, mais concerne des textes ou discours de domaines divers, publicitaires, narratifs, littéraires, fictifs. Dans chaque situation, l'examen de l'ethos permet de prendre la mesure de l'adhésion de l'interlocuteur liée, non pas à la force de conviction des arguments et des idées avancées, mais à l'image de soi que projette le locuteur qui veut convaincre l'interlocuteur d'adhérer à «une certaine position discursive». (Maingueneau,1999, p. 76)

Dans ce dossier, nous nous intéressons plus spécifiquement à l'ethos biographique, à partir d'un corpus qui inclut la biographie en tant que telle et, d'une manière extensive, les formes qui empruntent au biographique ${ }^{2}$. La biographie connaît, depuis quelques décennies, un renouvellement qui a entraîné l'apparition de formes différentes, brouillant les frontières entre la réalité historique et l'univers fictif, entre le récit et l'essai, entre le biographique et l'autobiographique et proposant des formes hybrides dont on pourrait mentionner, pour exemple, la biographie imaginaire ${ }^{3}$. En réponse à cette effervescence créative, la recherche

\footnotetext{
2 Les textes du présent dossier ont d'abord été l'objet de communications présentées au colloque « Modalités de l'ethos biographique » qui s'est tenu à l'Université du Québec à Trois-Rivières, les 8-9 mai 2007, dans le cadre du $75^{\mathrm{e}}$ congrès de l'ACFAS. Le projet de ce colloque s'inscrivait dans le cadre de ma recherche postdoctorale subventionnée par le FQRSC ainsi que dans le cadre plus large d'une recherche sur Les postures du biographe subventionnée par le CRSH et codirigée par Frances Fortier (UQAR) et Robert Dion (UQAM). Mes remerciements vont à tous ces organismes et personnes par qui ce projet a été rendu possible.

${ }^{3}$ Le récent ouvrage collectif Vies en récit. Formes littéraires et médiatiques de la biographie et de l'autobiographie (Robert Dion et al. 2007) donne une idée de ce renouvellement.
} 
emprunte des avenues qui réfléchissent sur différents enjeux liés à l'utilisation de l'archive, à l'équilibre entre vérité et fiction, ou encore, aux modalités de la relation entre la vie et l'œuvre dans le cas des biographies d'écrivain. Mais il est également un autre aspect qui stimule la recherche dans ce domaine: il s'agit de l'intérêt que suscite l'écriture biographique lorsque l'on s'arrête à la relation qui se tisse entre ces deux instances que sont le biographe et son biographé. Examinée sous cet angle, la biographie laisse voir le travail réflexif du biographe qui se sait mobilisé dans une entreprise où il tient, certes, un rôle de narrateur, mais où il est aussi un participant à part entière engagé dans une relation qui l'oblige à s'interroger sur la distance qu'il fonde entre lui et le biographé. Ainsi, Frédéric Regard voit dans le discours de la biographie littéraire qui se bâtit autour de la relation entre l'écrivain biographe et l'écrivain biographé une "pratique éminemment intersubjective ». (p. 26) Dans le même ordre d'idée, Martine Boyer-Weinmann juge que le biographe, inscrit dans un « cheminement dialogué », ne peut pas «se dérober au travail de la relation, c'est-à-dire de la différence». (p. 103-104) En fait, nombreuses sont les œuvres qui soulèvent la question du rapport entre les deux instances et la problématisation de cette relation semble constituer l'un des ancrages de la réflexion sur les écritures biographiques contemporaines. C'est dans ce contexte, par exemple, que Frances Fortier montre «la dimension essentiellement relationnelle» (p. 62) de la biographie d'écrivain qui se pose comme revendication de filiation. D'aucuns s'interrogent sur l'indistincte frontière qui sépare le biographique de l'autobiographique dans ces œuvres hybrides qui semblent tenter de "ressaisir ce qui de l'autre entre dans la constitution du moi » (Viart, p. 18), ou encore, de 
démontrer que l'auteur compose, «avec et par ce détour vers autrui, l'irréductible maintien d'une individualité ». (Rabaté, p. 44)

Cheminement dialogué, pratique intersubjective, revendication de filiation, démarche réflexive établie à la faveur d'un détour vers autrui, le projet biographique présente donc un espace relationnel où le rapport du biographe à son biographé se joue à la fois du côté d'une rencontre qui peut être plus ou moins fusionnelle et d'un exercice de différentiation, mais où s'énonce constamment la tension entre le je et le $i l$, entre le soi et l'autre. Cette tension identitaire, même si elle semble davantage associée aux biographies contemporaines, caractérise cependant toute écriture biographique qui, à travers la représentation de cette relation entre le biographe et le biographé, se donne à la fois comme un discours sur l'autre et une énonciation de soi. Or, la notion d'ethos permet d'appréhender la construction de l'image de soi du biographe en tenant compte de ce double objectif. D'abord, dans la relation d'interlocution qui le noue au lecteur, le biographe, au premier chef, présente le biographé. De ce point de vue, l'énonciation produit un ethos qui doit en quelque sorte légitimer la prise de parole du biographe, établir sa crédibilité face au lecteur et ainsi conduire ce dernier à adhérer à la représentation du biographé que lui présente le biographe. Comme l'écrit Ruth Amosssy, «le biographe entreprend une reconstitution qui corrobore son interprétation particulière du réel.» (2001, p. 162) À cet objectif premier de quête de l'adhésion du lecteur au propos que le biographe tient sur un tiers, il y a également cette énonciation de soi que le biographe construit. La reconnaissance de la singularité de l'autre, aux fondements de la relation biographique, engage le biographe à 
sonder sa singularité, à faire un retour sur ses propres interrogations. Là aussi, la notion d'ethos s'avère pertinente puisque la construction de l'ethos est liée à la question de l'identité et concerne, comme le note Maingueneau, «la stratégie de parole d'un locuteur qui oriente le discours de façon à se façonner à travers lui une certaine identité. » (2002, p. 5) Si l'ethos peut prendre appui sur certaines régularités liées à des genres du discours et adopter une forme plus attendue, il peut être renouvelé puisque le locuteur est en mesure de moduler à son gré son image discursive pour influer sur les attitudes, les opinions de l'allocutaire et ainsi se démarquer et insister sur sa propre singularité. Cette construction, comme le souligne Amossy, est ce qui va permettre « de créer un rapport nouveau à soi et à l'autre. » (2006,, p. 96) C'est ainsi que dans les écritures biographiques, l'image de soi du locuteur construite à travers un ensemble de signes à destination de l'interlocuteur donne accès à la singularité du locuteur dans sa fonction de biographe, mais rejoint également d'autres aspects de l'identité du locuteur, laissant place, par exemple, à l'émergence de la figure de l'écrivain.

Le corpus de la biographie, d'une grande diversité de formes, de tons, d'objectifs visés, offre ainsi l'occasion d'une exploration des modalités de l'ethos biographique et de sa variabilité, selon la nature du projet du biographe (biographe écrivain, biographe historien, biographe témoin, biographe fictif), ou selon son degré d'implication (biographe accusant la distance que donne la rigueur du discours savant, biographe «biophage, dévoré par son transport vers l'autre». (Dosse, p. 130) Les six textes de ce dossier répondent à cette diversité du corpus par une diversité dans les approches proposées et dans les questions soulevées. Ainsi la singularité de la démarche du 
biographe face à son biographé ressort d'une façon particulière dans les articles de David Vrydaghs et de Frances Fortier qui, chacun, explorent l'ethos de plusieurs biographes d'un même auteur. La réflexion de Vrydaghs se situe résolument dans le cadre d'une approche qui prend en compte la dimension sociologique de la notion d'ethos. Mettant de l'avant le fait que le genre biographique constitue un genre phare dans tout processus d'assignation d'une identité littéraire à un écrivain, Vrydaghs s'intéresse au rôle du biographe dans la construction de l'image de l'écrivain biographié, notamment par l'ethos que le biographe adopte et qui influe sur le degré d'adhésion du public lecteur face au récit et au portrait d'écrivain qui lui est proposé. Prenant pour objet d'observation deux biographes d'Henri Michaux, Vrydaghs fait état des entorses au genre que font subir Jean-Pierre Martin et Robert Bréchon pour arriver à écrire leur biographie, le premier alternant entre l'ethos du romancier et l'ethos du critique littéraire, alors que Bréchon sacrifiera en quelque sorte le projet biographique en optant pour se présenter à travers l'ethos d'un critique, mais aussi l'ethos d'un ami qui respecte le souhait même de l'écrivain Michaux de vouloir garder le secret sur sa vie privée. Par la mise en parallèle de l'autobiographie de Gabrielle Roy et des biographies d'André Vanasse et de François Ricard parues ultérieurement, Frances Fortier, donne également la mesure de la diversité des ethos. Si Gabrielle Roy a choisi de délaisser les éléments factuels de sa vie pour insister sur la quête d'une vérité essentielle, tentant de se montrer sous un jour "aimable », la proposition de Ricard incarne l'ethos du biographe sérieux, impartial, qui met le savoir objectif au service de la narration de la vie du biographé et donc qui sait se rallier le lectorat par son autorité en la matière, alors que 
Vanasse propose l'ethos du témoin intime et privilégié qui s'approche d'une quasi autobiographie tant il se met au premier plan.

Ces deux articles montrent bien que l'ethos que choisit d'adopter le biographe conditionne sa façon de raconter la vie du biographé. Mais si l'écriture biographique constitue le récit de la vie d'un tiers, elle est également concernée par la relation qui se noue entre le biographe et son biographé, relation qui informe sur le degré d'implication du biographe. Michel Magniez aborde cet aspect de l'écriture biographique et montre comment la biographie de Cicéron qu'écrit Stefan Zweig va permettre à ce dernier «de tracer obliquement le portrait de ses propres convictions » et de proposer une présentation de soi au miroir de l'autre, tout en exhibant l'ethos du biographe sérieux qui s'appuie sur la validité de l'archive et donc émet un discours qui s'en tient à la vérité historique.

Certaines œuvres contemporaines, notamment celles qui intègrent une part d'imaginaire, se situent plus ostensiblement du côté de l'écriture de soi et se maintiennent volontairement à la frontière du biographique et de l'autobiographique, du réel et de sa transposition. C'est ce corpus qu'abordent Caroline Dupont et Maryse Aubut à travers les œuvres de Victor-Lévy Beaulieu et de Jean-Benoît Puech. On assiste, dans ces deux cas, à une sorte de détournement du projet biographique, la transgression de la biographie conventionnelle visant foncièrement à la mise en œuvre d'une écriture. Dupont examine chez Beaulieu l'invention d'un double fictionnel, Abel Beauchemin, personnage et narrateur-biographe. Elle va donc observer l'ethos de ce personnage fictif et montrer comment la construction stratégique d'un ethos de l'humilité - susceptible 
de susciter l'adhésion du lecteur - qui glisse graduellement vers un ethos d'usurpateur va laisser émerger une forme inversée de la biographie où, au bout du compte, se donne à lire l'autobiographie de Beauchemin/Beaulieu sous l'éclairage de l'œuvre du biographé, qu'il se nomme Herman Melville ou James Joyce. Maryse Aubut s'intéresse, pour sa part, à la présence d'un double imaginaire qui prend le statut d'auteur de l'ouvrage. C'est sous la signature de Benjamin Jordane que JeanBenoît Puech, dans L'apprentissage du roman, fait le récit - mais en utilisant des noms d'emprunts - de la naissance de sa vocation d'écrivain et du rôle qu'y a tenu l'écrivain-mentor Louis-René des Forêts. Aubut explore cette situation où Puech, qui attribue la paternité de son propre journal à ce double imaginaire, va se refuser d'adopter l'ethos du biographe de Jordane, pour choisir de se présenter auprès du lectorat, à titre de critique, commentateur ou lecteur de l'œuvre de Jordane, donc confiné, comme elle le précise, "dans une parole de la répétition", devenant, selon les mots mêmes de Puech, «l'auteur de l'auteur».

La dernière partie du dossier s'attache plus précisément aux marges du biographique. Béatrice Fleury et Manon Auger proposent d'examiner les modalités de l'ethos, d'une part, dans l'acte de réception d'une œuvre testimoniale par les membres d'une collectivité, et d'autre part, dans les écrits autobiographiques que constituent les journaux d'écrivains. La construction de l'ethos, avons-nous dit, demeure liée à la question de l'identité et ces deux articles explorent plus particulièrement cet aspect. Béatrice Fleury s'intéresse à l'ethos d'une collectivité dans une situation de communication précise, à travers un corpus constitué des courriers de vingt-cinq personnes ayant réagi au documentaire Mémoires d'immigrés. 
L'héritage maghrébinde Yamina Benguigui. Fleury expose les différentes modalités - spéculaire, historisée, sensible, pacifiée - de l'ethos biographique des spectateurs-écrivants dans la situation d'échange qui le détermine, c'est-à-dire dans sa relation à l'ethos biographique de la réalisatrice, tel qu'exposé dans le documentaire. Manon Auger, pour sa part, explore l'ethos diaristique dans certains journaux d'écrivains, journaux qui, dès le départ sont destinés à être lus et publiés. Ses observations montrent que l'écriture diaristique est influencée par le type de conscience qu'a l'auteur de son statut d'écrivain, l'écriture du journal semblant être perçue, pour certains d'entre eux, comme difficilement assimilable à leur propre pratique d'écriture. L'examen des parcours proposés permet de mettre en évidence les différentes façons de négocier des aménagements entre l'ethos du diariste et l'ethos de l'écrivain, selon un équilibre propre à chaque écrivain-diariste et selon la «volonté plus ou moins affirmée d'assurer la cohésion de la figure de l'écrivain. »

\section{Bibliographie}

Amossy, Ruth, (dir.). 1999, Images de soi dans le discours. La construction de l'ethos, Lausanne, Delachaux et Niestlé, p. 930 .

—. 2001, "Images de soi, images de l'autre dans l'interaction (auto)biographique: La mort est mon métier de Robert Merle » Revue des sciences humaines, no 263, p. 161-182.

- . 2006, L'argumentation dans le discours, Paris, Armand Colin, coll. « cursus». 
BOYER-WEINMANN, Martine. 2005, La relation biographique, Seyssel, Champ Vallon.

Dion, Robert, Frances ForTIER, Barbara HAVERCROFT et HansJürgen LÜSEBRINK (dir.). 2007, Vies en récit. Formes littéraires et médiatiques de la biographie et de l'autobiographie, Québec, Nota bene.

DosSE, François. 2005, Le pari biographique. Écrire une vie, Paris, La Découverte.

FORTIER, Frances. 2005, «La biographie d'écrivain comme revendication de filiation: médiatisation, tension, appropriation », Protée, vol. 33, n³, p. 51-64.

MaingueneaU, Dominique. 1999, «Ethos, scénographie, incorporation », dans Ruth Amossy (dir.), Images de soi dans le discours. La construction de l'ethos, Lausanne, Delachaux et Niestlé, p. 75-100.

—. 2002, «Problèmes d'ethos », Pratiques, no 113-114, p. 55-67.

RABATÉ, Dominique. 2003, « Ce qui n'a pas de témoin? Les vies imaginaires dans l'écriture contemporaine », dans Rosa Galli Pelligrini (dir.), Stratégies narratives 2. Le roman contemporain, Paris, Presses de l'Université de ParisSorbonne, p. 29-44.

REGARD, Frédéric. 1999, « Les mots de la vie : introduction à une analyse du biographique », dans Frédéric Regard (dir.), $L a$ biographie littéraire en Angleterre (XVIIe - XXe siècles). Configurations, reconfigurations $d u$ soi artistique, SaintÉtienne, Publications de l'Université de Saint-Étienne, p. 1130 .

VIART, Dominique. 2001, «Dis-moi qui te hante. Paradoxes du biographique », Revue des sciences humaines, $n^{\circ} 263$ (juilletseptembre), p. 7-33. 


\section{Résumé}

Les textes regroupés dans ce dossier explorent les modalités de l'ethos biographique. Il nous apparaît que l'œuvre biographique, caractérisée par la relation singulière qui s'établit entre le biographe et le biographé, offre un contexte particulier à la construction de l'ethos, puisque l'écriture s'y propose à la fois comme discours sur l'autre et énonciation de soi. Les diverses analyses proposées montrent que l'image de soi construite à travers un ensemble de signes à destination de l'interlocuteur donne accès à la singularité du locuteur dans sa fonction de biographe, mais révèle également d'autres aspects de l'identité de ce dernier, laissant place, entre autres, à l'émergence de la figure de l'écrivain.

\section{Abstract}

The texts in this issue explore the modalities of the biographical ethos. They show that biographical writing, which is characterized by the peculiar relationship that is established between biographer and biographee, offers a context for constructing a specific ethos, since the writing offers both discourse on another person and self-enunciation. The various analyses demonstrate that the self-image constructed from the set of signs aimed at the interlocutor reveal the locutor's singularity in his role of biographer, but also reveal other aspects of his identity, and allow, among other things, the emergence of the writer's image. 